Pulsars : Problems \& Progress

ASP Conference Series, Vol. 105, 1996

S. Johnston, M. A. Walker and M. Bailes, eds.

\title{
On Galactic magnetic field derived from RMs of pulsars
}

\author{
J.L. Han
}

Beijing Astronomical Observatory, CAS, Beijing 100080, China

G.J. Qiao

Department of Geophysics, Peking University, Beijing 100871, China

\begin{abstract}
RMs of all 262 pulsars are used to see the large scale magnetic field of our Galaxy. We find that: (1). RMs of 91 pulsars at high galactic latitude $\left(|b|>8.6^{\circ}\right)$ show two toroidal magnetic rings antisymmetric with respect to galactic plane. These data shouldn't be used for modelling the large scale field in Galactic disk; (2). pulsars at low galactic latitude are concentrated on the spiral arms; (3). the directions of fields have a similar pitch angle to that of spiral arms.
\end{abstract}

The best way to reveal magnetic field of our Galaxy is to study the Faraday rotation of pulsars (Manchester 1974). Earlier efforts have concentrated on model-fits of Rotation Measures (RMs) of some 200 pulsars, by assuming the electron distribution and estimating pulsar distances (eg. Han \& Qiao 1994). As shown by Han \& Qiao (1994), more RMs of sourthen pulsars are very necessary to overcome the selection effect of the sample. Recently, new measurements of RMs of 62 pulsars have been published (Rand \& Lyne 1994, Qiao et al. 1995), and 45 of them in the sourthen sky.

Taking the pulsar distances from Taylor, Manchester \& Lyne (1993), we project all available 262 pulsars with measured RMs onto the Galactic plane. It could be puzzling to understand the positive and negative RM data in the vicinity of the Sun, within $1.5 \mathrm{kpc}$, since it is impossible to get a reasonable direction of local magnetic field from the RMs of these nearby pulsars.

Recently, Han et al. (1995) noticed that the RMs of extragalactic radio sources show antisymmetric toroidal magnetic fields with respect to the Galactic plane. The feature could be induced by toroidal magnetic rings above and below the Galactic plane, within which the fields have opposite directions. We note that the RMs of 91 pulsars at high latitude $|b|>8.6^{\circ}$ (figure omitted) show the same antisymmetric magnetic rings.

It is thus realized immediately that pulsars at high galactic latitude should be excluded from the sample if the magnetic field in the Galactic disk is modelfitted from the RMs of pulsars. Therefore, only 171 pulsars at low galactic latitude $\left(|b|<8.6^{\circ}\right)$ are suitable for this purpose. As can be seen from Fig.1:

(1). First, these pulsars concentrate on the spiral arms delineated by $\mathrm{O}-\mathrm{B}$ stars (Lynds 1980) and molecular clouds (Solomon \& Rivolo 1989). Such a concentration is more clearly to be seen from Fig. 1 than from high latitude pulsars. 


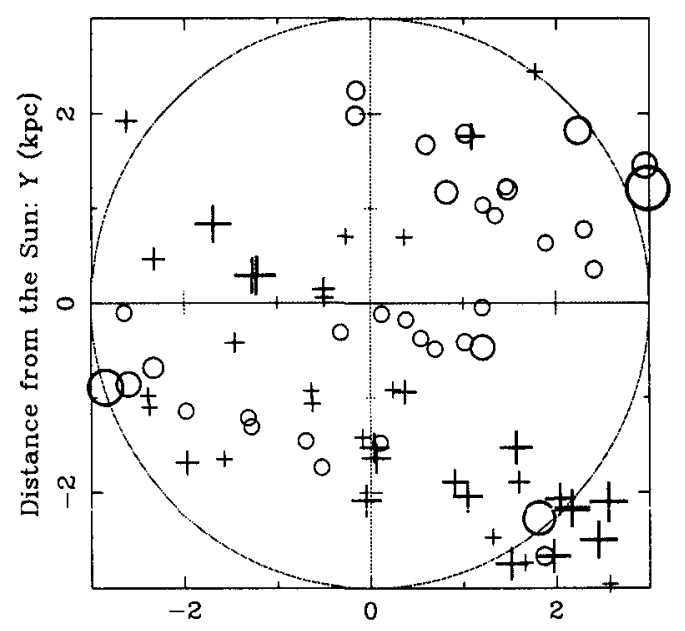

Distance from the Sun: $X(k p c)$

Figure 1. The RMs of nearby pulsars at low latitude $\left(|b|<8.6^{\circ}\right)$ projected to the Galactic plane. Circles indicate negative RMs, and + positive ones.

(2). These RMs are qualitatively consistent well with the model of Han \& Qiao (1994) for the magnetic field in the Galactic disk.

(3). The magnetic field in the Orion arm points towards $l \simeq 80^{\circ}$ and it belongs to the same "magnetic arm" of Perseus arm in the BiSymmetric Spiral field model of Han \& Qiao (1994). It suggests that the Orion arm should bifurcate from the Perseus arm.

Acknowledgments. We thank National Nature Science Foundation of China for finacial support. JLH also acknowledges the supports from the Science Foundation for Young Researchers of CAS, the Su-Shu Huang Astrophysics Research Foundation of CAS and Director Foundation of BAO.

\section{References}

Han, J.L., et al., 1995, in preparation.

Han, J.L., Qiao, G.J., 1994, A\&A, 288, 759

Lynds, B.T., 1980, AJ, 85(8), 1046

Manchester, R.N., 1974, ApJ, 188, 637

Qiao, G.J., Manchester, R.N., Lyne, A.G., Gould, D.M., 1995, MNRAS, 274, 572

Rand, R.J., Lyne, A.G., 1994, MNRAS, 268, 497

Solomon, P.M., Rivolo, A.R., 1989, ApJ, 339, 919

Taylor, J.H., Manchester, R.N., Lyne, A.G., 1993, ApJS, 88, 529 